\title{
Spectrum of the seismic-electromagnetic and acoustic waves caused by seismic and volcano activity
}

\author{
S. Koshevaya ${ }^{1}$, N. Makarets $^{2}$, V. Grimalsky ${ }^{3}$, A. Kotsarenko ${ }^{4}$, and R. Perez Enríquez ${ }^{4}$ \\ ${ }^{1}$ Universidad Autonoma del Estado de Morelos (UAEM), CIICAp, Cuernavaca, Mexico \\ ${ }^{2}$ Kyiv National Shevchenko University, Faculty of Physics, Kyiv, Ukraine \\ ${ }^{3}$ Instituto Nacional de Astrofisica, Optica y Electronica (INAOE), Puebla, Mexico \\ ${ }^{4}$ Centro de Geociencias, Juriquilla, UNAM, Querétaro, Mexico
}

Received: 30 June 2004 - Revised: 24 November 2004 - Accepted: 7 December 2004 - Published: 2 February 2005

Part of Special Issue "Precursory phenomena, seismic hazard evaluation and seismo-tectonic electromagnetic effects"

\begin{abstract}
Modeling of the spectrum of the seismoelectromagnetic and acoustic waves, caused by seismic and volcanic activity, has been done. This spectrum includes the Electromagnetic Emission (EME, due to fracturing piezoelectrics in rocks) and the Acoustic Emission (AE, caused by the excitation and the nonlinear passage of acoustic waves through the Earth's crust, the atmosphere, and the ionosphere). The investigated mechanism of the EME uses the model of fracturing and the crack motion. For its analysis, we consider a piezoelectric crystal under mechanical stresses, which cause the uniform crack motion, and, consequently, in the vicinity of the moving crack also cause non-stationary polarization currents. A possible spectrum of EME has been estimated. The underground fractures produce Very Low (VLF) and Extremely Low Frequency (ELF) acoustic waves, while the acoustic waves at higher frequencies present high losses and, on the Earth's surface, they are quite small and are not registered. The VLF acoustic wave is subject to nonlinearity under passage through the lithosphere that leads to the generation of higher harmonics and also frequency downconversion, namely, increasing the ELF acoustic component on the Earth's surface. In turn, a nonlinear propagation of ELF acoustic wave in the atmosphere and the ionosphere leads to emerging the ultra low frequency (ULF) acoustogravity waves in the ionosphere and possible local excitation of plasma waves.
\end{abstract}

\section{Introduction}

Investigations of the energy flow mechanisms from the lithosphere into the atmosphere and the ionosphere caused by natural hazards, like seismic and volcano activity, for example experiment MASSA (Galperin, 1985), are very important,

Correspondence to: S. Koshevaya

(svetlana@uaem.mx) because they give information on the development of seismic and volcanic activity.

The energy flow mechanisms possess different precursors (Warwick, 1963), due to the electromagnetic, geochemical, and acoustic basic channels of the lithosphere-ionosphere coupling (Gokhberg et al., 1995; Molchanov et al., 1995; Tarantsev and Birfeld, 1973; Kotsarenko et al., 1997). The geochemical channel consists of the release of various gases, such as radioactive gases, aerosols, metal ions etc., from the area of mutual touches and deformations of the tectonic plates (Aki and Richards, 1980).

- The chemical channel can be described as the formation of clouds, a spatial distribution of polar lights, a cross distribution of the ionosphere plasma density in D-, E-, and F-layers, an appearance of abnormal propagation bands of radio waves, etc. One of the basic results of this mechanism of coupling is the increase of the conductivity of the atmosphere and the ionosphere above the places of fracture of tectonic plates.

- The electromagnetic channel can be described as follows. In the epicenters of preparing earthquakes, alternating electric currents $\delta \boldsymbol{j}(\boldsymbol{r}, t)$ appear. In accordance with the Maxwell's equations, they are the sources of alternating electromagnetic fields, which reach the ionosphere practically instantly and create over there an electromagnetic response. The main problem is to determine $\delta \boldsymbol{j}$, related to the seismic processes. It is possible to define some mechanisms (Surkov et al., 2003), which produce the occurrence of the alternating currents in places of tectonic plate fracturing, such as: piezoeffect, electro kinetic effect, the motion of charged dislocations, the motion of underground Earth water with the consequent increase of the electric conductivity, etc. With the knowledge of the spatial - temporal current distribution and the ionosphere conductivity tensor, it is 


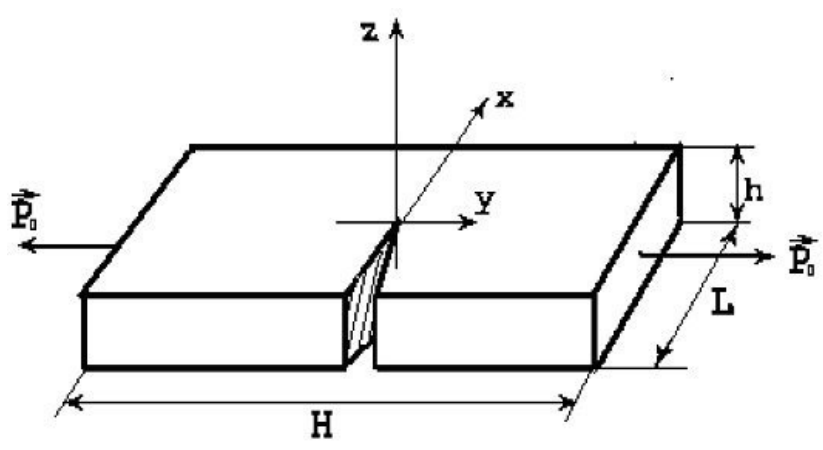

Fig. 1. The crack for the simple case of quartz, the optical axis is oriented in the $Z$ direction and the magnetic moment caused by circular vortical polarization current in the plane of the crack is oriented along the same axis. Here $P_{0}$ is the mechanical stress (Makarets, 2002).

possible to determine the electromagnetic response of the ionosphere due to the current pulse originated in the lithosphere. This problem was solved by Molchanov and Hayakawa (Molchanov et al., 1995).

The acoustic channel of the lithosphere-ionosphere coupling seems quite effective (Tarantsev et al., 1973), due to atmospheric acoustic waves excited by fluctuations of the terrestrial surface. This channel manifests in different phenomena, for example, the plasma wave excitation and the periodic structure in the ionosphere, increasing transparency for radio waves, linear and nonlinear generation of magnetic perturbations, oscillations of E- and F-layers in the ionosphere caused by acoustic and acoustic-gravity waves, as well as the nonlinear transformation of the wave spectrum in the atmosphere, ionosphere, and the lithosphere (Kotsarenko et al., 1997). The electromagnetic and acoustic channels are presented below as examples of EME and $\mathrm{AE}$, which were experimentally observed. There are a lot of papers devoted to the acoustic mechanism of coupling (Hayakawa and Molchanov, 2002; Hines, 1974; Hayakawa and Fujinawa, 1994; Molchanov et al., 2001). But the main attention in this paper is devoted to a comparison of the EME and AE spectra. The deep fracturing in rocks produces only VLF, ELF, and ULF EM spectra because high losses take place in the lithosphere. The nonlinear interaction of acoustic waves in the lithosphere, the atmosphere, and the ionosphere also leads to the release of the lower frequency part of the spectrum, as it has been demonstrated.

\section{Modeling of the electromagnetic emission}

We consider a piezoelectric crystal of symmetry 32 under mechanical stresses, which causes the uniform motion of the crack (Makarets et al., 2002). The direction of the crack motion and the orientations of the front of the crack are analyzed as arbitrary, in a relation to the crystal symmetry axes (see Fig. 1). Non-stationary mechanical stresses in the vicinity of the moving crack cause non-stationary polarization cur-

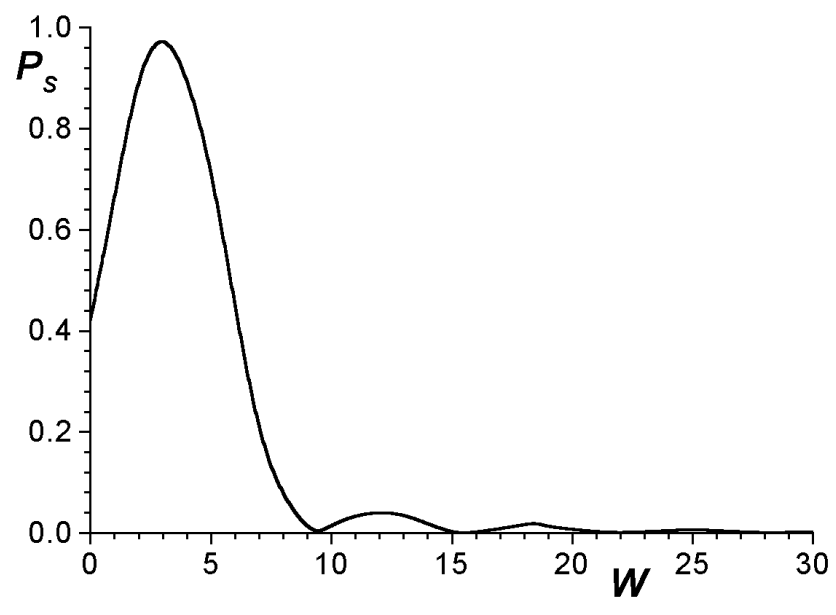

Fig. 2. The dependence of the power of seismo-electromagnetic wave $P_{S}$ (relative units, connected with the ratio of the radiation energy to the mechanical energy $P_{S}=\frac{P e}{P m}$ ) caused by the single crack in the quartz on the undimensional frequency $w=\omega \frac{L}{V}$.

rents. These currents generate electromagnetic waves. The first kind of current is the electric polarization current, $I_{P}$, and the second one is the effective magnetization current $I_{M}$, such that $I_{P} / I_{M} \approx(V / c)^{2} \ll 1$, where $V$ and $c$ are the crack velocity and the velocity of light, respectively. Using the Maxwell's equations and classical equations of the elasticity theory, and taking into account the definition of the deformation tensor $2 U_{k l}=\nabla_{l} U_{k}+\nabla_{k} U_{l}$, and also using the crystallographic coordinate system, the equations for the electric field $\boldsymbol{E}(\boldsymbol{r}, t)$ and the mechanic displacement $\boldsymbol{U}(\boldsymbol{r}, t)$ can be obtained (Landau and Lifshitz, 1960; Maugin, 1988):

$$
\begin{aligned}
& \Delta \boldsymbol{E}-\nabla \operatorname{div} \boldsymbol{E}-\frac{\varepsilon_{i j}}{c^{2}} \frac{\partial^{2} E_{j}}{\partial t^{2}} \boldsymbol{e}_{i}=\frac{1}{\varepsilon_{0} c^{2}} e_{i k l} \frac{\partial^{2} U_{k l}}{\partial t^{2}} \boldsymbol{e}_{i}, \\
& c_{i j k l} \frac{\partial U_{k l}}{\partial x_{j}}-\rho \frac{\partial^{2} U_{i}}{\partial t^{2}}=e_{k i j} \frac{\partial E_{k}}{\partial x_{j}},
\end{aligned}
$$

where $U_{i j}=\frac{1}{2}\left(\frac{\partial U_{i}}{\partial x_{j}}+\frac{\partial U_{j}}{\partial x_{i}}\right)$ are the components of the deformation tensor; $c$ is the velocity of light in vacuum; $c_{i j k l}, \quad \varepsilon_{i j}, \quad e_{i j k}$ are the components of the elastic, dielectric, and piezoelectric tensors of the crystal, respectively; $\boldsymbol{e}_{i}$ are unit vectors of the crystallographic system; $\rho$ is density of the crystal; $\varepsilon_{0}$ is electric constant; and the indices are $i, j, k, l=1,2,3$. The Eqs. (1) and (2) are written in SI units, hence the units of the material tensors $c_{i j k l}$ and $e_{i j k}$ are $\mathrm{Pa}$ and $\mathrm{C} / \mathrm{m}^{2}$, respectively. The conductivity in piezoelectric medium is neglected here.

In order to find the solution of these equations, in addition to the mentioned assumptions, some boundary conditions should be considered for the electric field, the deformations on the crystalline surfaces and on the new surfaces created by the crack. However, exact solutions of Eqs. (1) and (2) with such boundary conditions are still unknown for any kind of crystals, therefore we should use some approximations. The analytical results of the problem are the following 
Table 1. Frequencies of electromagnetic emission caused by cracks of different sizes. The velocity of the crack is $V=1000 \mathrm{~m} / \mathrm{s}$.

\begin{tabular}{cc}
\hline $\begin{array}{c}\text { Maximum length of } \\
\text { size crack, }(\mathrm{cm})\end{array}$ & $\begin{array}{c}\text { Frequency } \omega, \\
\left(10^{3} \mathrm{~s}^{-1}\right)\end{array}$ \\
\hline 0.1 & 10 \\
1 & 1 \\
10 & 0.1 \\
100 & 0.01 \\
\hline
\end{tabular}

(see Makarets et al., 2002 for details). For a single crack, the intensity of the electromagnetic emission has the following dependence:

$P_{\text {sin } g l e}=w^{2} y^{5} \frac{\sin ^{2}\left(\frac{w y}{2}\right) \sin ^{2}(w \beta \cos (\vartheta))}{\cos ^{2}(\vartheta)} \sin ^{4}(\vartheta)$,

where $w$ is the dimensionless frequency, related to the frequency $\omega$ by $w=\omega \frac{L}{V}, L$ is the maximum size of the crack; $\beta=V / S_{2} ; S_{2}$ the velocity of the transverse acoustic wave, $y$ is the dimensionless size of the crack expressed in terms of $L, \vartheta$ is the angle in the coordinate system of the direction of the propagation of the EM radiation with the axis OZ parallel to the optical axis of the piezoelectric crystal.

With $y=1$ and $\vartheta=\pi / 2$, we have plotted the EME power generated by the single crack in the piezoactive plane, as a function of $w=\omega \frac{L}{V}$, where $V$ is the crack velocity (see Fig. 2 and Table 1). The parameters of crystalline quartz have been used. If fracturing by seismic processes takes place on the Earth's surface, volcano activity or during impact events (Khavroshkin et al., 2001; Musatenko et al., 1997; Berezhnoy et al., 2002), a wide spectrum, that includes also radio waves, has been observed.

In the case of deep fracturing (the thickness of the covering soil is about $1 \mathrm{~km}$, its conductivity is about $10^{-2} \mathrm{~S} / \mathrm{m}$ ), a strong dissipation of electromagnetic waves at frequencies above 3-10 kHz occurs (Molchanov et al., 2001). Thus, only the lower part of electromagnetic spectrum may be observed. This part of the radiation corresponds to the sizes of the moving cracks within the piezoelectric rocks larger than $1 \mathrm{~cm}$.

\section{Modeling of acoustic emission}

\subsection{Nonlinear passage through the lithosphere}

The geometry of the model is shown in Fig. 3. The cylindrical symmetry of the source of underground seismic explosion (or plate deformation) is assumed. The acoustic waves are excited under seismic processes as a burst-like envelope of a finite transverse scale. Then the excited waves pass through the Earth's lithosphere. It is possible to use the elasticity theory with nonlinear modules and to take into account damping and diffraction of the waves. The equations of the elasticity theory, in the case of the Earth's crust as an isotropic

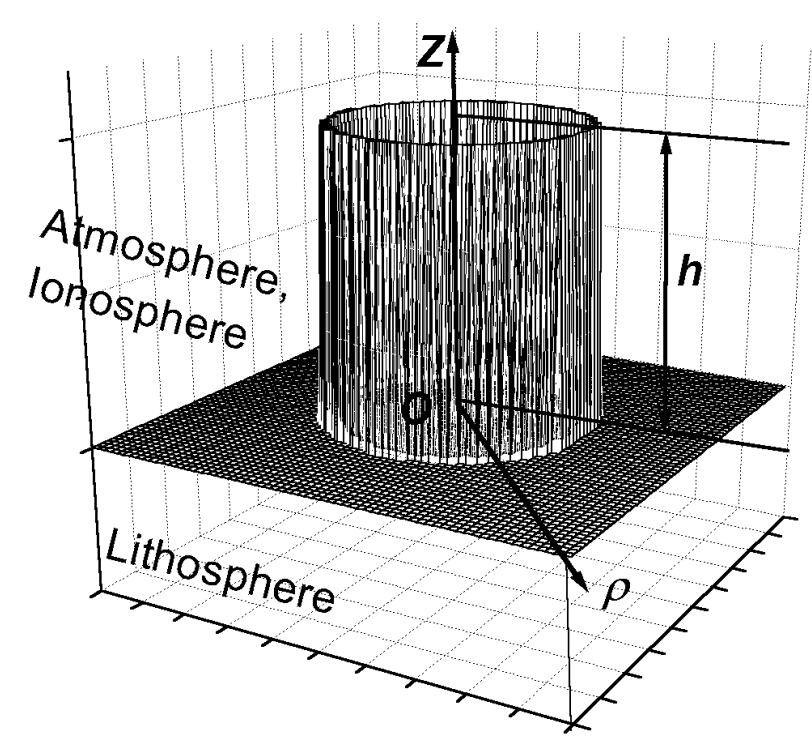

Fig. 3. Geometry of the nonlinear passage of the acoustic waves caused by underground fracturing through the lithosphere, the atmosphere, and the ionosphere.

medium, possess the following form for the mechanic displacement:

$$
\begin{aligned}
& \frac{\partial^{2} \boldsymbol{U}}{\partial t^{2}}=\nabla\left\{\left(S_{l}^{2}-S_{t}^{2}\right) \cdot \operatorname{div} \boldsymbol{U}\right\} \\
& +S_{t}^{2} \cdot \Delta \boldsymbol{U}+\Gamma(z) \frac{\partial}{\partial t} \Delta \boldsymbol{U}+B(z) \nabla\left\{(\operatorname{div} \boldsymbol{U})^{2}\right\},
\end{aligned}
$$

where the coefficients $\Gamma(z)$ and $B(z)$ describe the viscosity and the nonlinearity coefficient of the elastic isotropic medium in cylindrical coordinate frame for the acoustic waves with the longitudinal and transverse velocities $S_{l, t}$, respectively. It is assumed that it is possible to neglect the derivatives of $S_{l, t}$. We take into account generally the dependence of the dissipation coefficient $\Gamma(z)$ on the coordinate. Rewrite the Eq. (4) by the components. For $z$-component of the displacement the equation is:

$$
\begin{aligned}
& \frac{\partial^{2} U_{z}}{\partial t^{2}} \cong\left(S_{l}^{2}-S_{t}^{2}\right) \cdot\left\{\frac{\partial^{2} U_{z}}{\partial z^{2}}+\frac{1}{\rho} \frac{\partial^{2}\left(\rho U_{\rho}\right)}{\partial z \partial \rho}\right\} \\
& +S_{t}^{2} \cdot\left\{\frac{1}{\rho} \frac{\partial}{\partial \rho}\left(\rho \frac{\partial U_{z}}{\partial \rho}\right)+\frac{\partial^{2} U_{z}}{\partial z^{2}}\right\}+ \\
& +\Gamma(z) \cdot \frac{\partial}{\partial t}\left(\frac{\partial^{2} U_{z}}{\partial z^{2}}\right)+B(z) \cdot \frac{\partial}{\partial z}\left(\frac{\partial U_{z}}{\partial z}\right)^{2} .
\end{aligned}
$$

Notice that the transverse profile is quite smooth. The transverse component of the elastic displacement is:

$$
\frac{\partial^{2} U_{\rho}}{\partial t^{2}} \cong\left(S_{l}^{2}-S_{t}^{2}\right) \cdot \frac{\partial^{2} U_{z}}{\partial \rho \partial z}+S_{t}^{2} \cdot \frac{\partial^{2} U_{\rho}}{\partial z^{2}} .
$$

Use the variables $z, \rho$, and $\eta=t-\int_{0}^{z} \frac{d z^{\prime}}{S_{l}\left(z^{\prime}\right)}$. Also the method of slowly varying profile is used so as this research is focused 


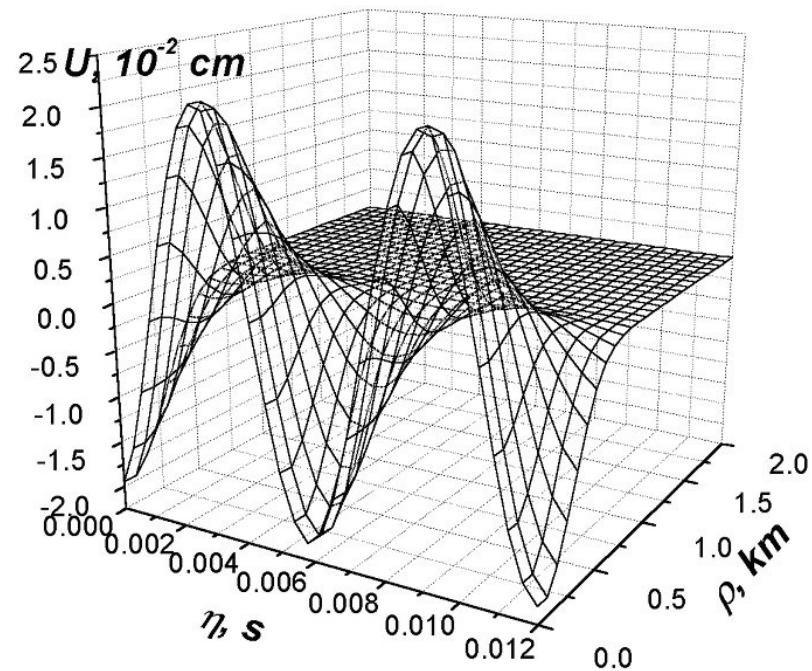

(a)

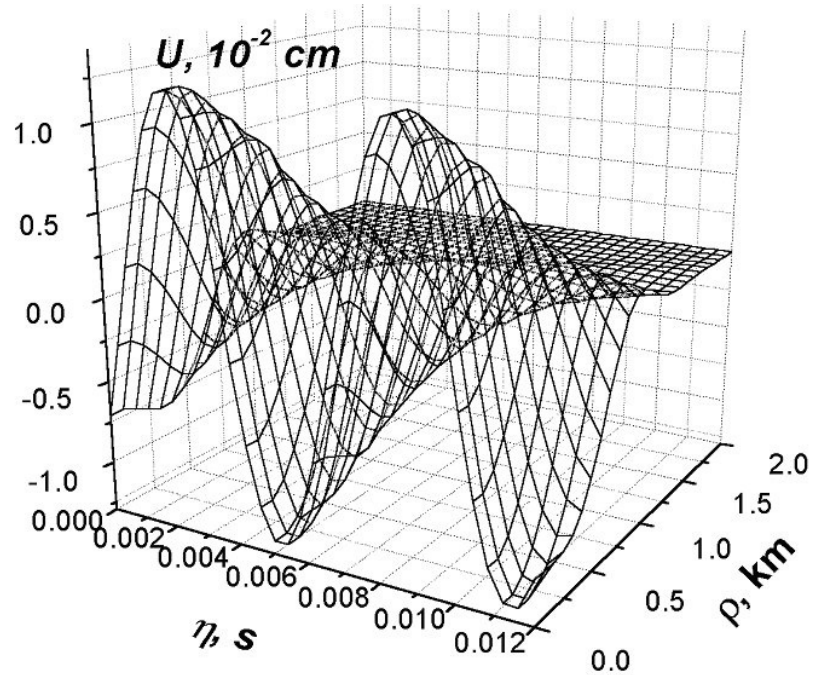

(c)

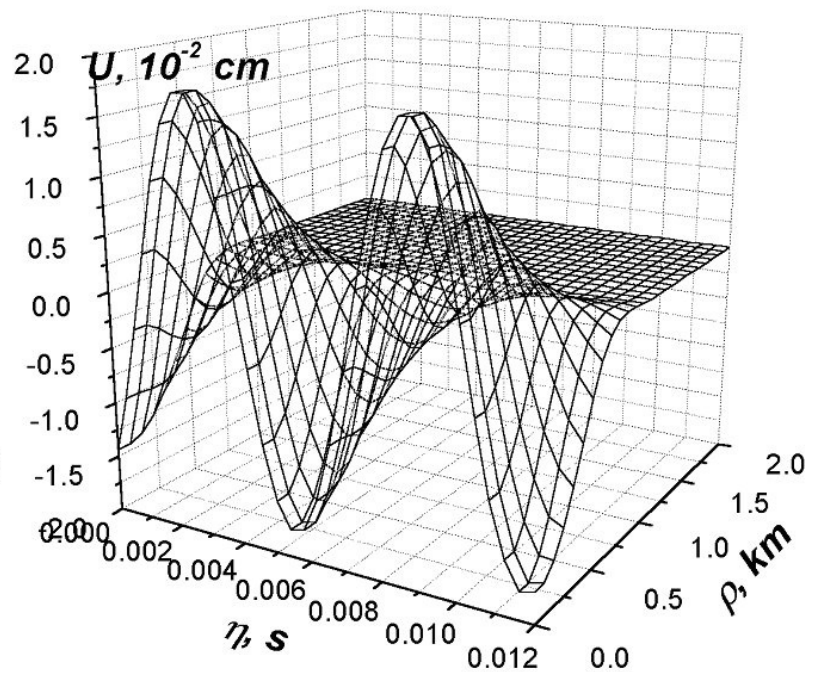

(b)

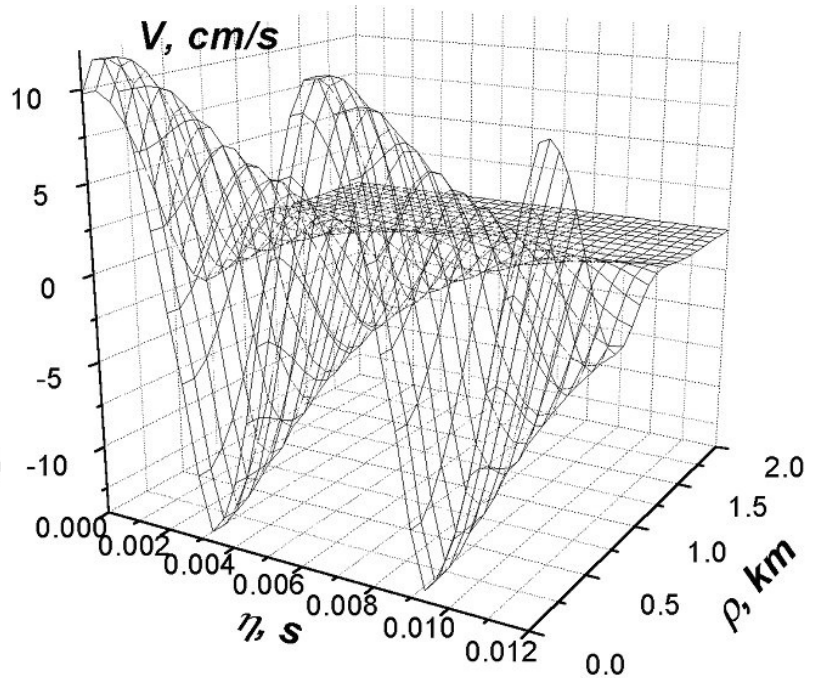

(d)

Fig. 4. The evolution of the nonlinearly excited ELF component of the mechanical displacement $U(\mathrm{~cm})$ in the lithosphere: (a) at $z=5 \mathrm{~km}$, (b) at $z=10 \mathrm{~km}$; (c) at $z=20 \mathrm{~km}$ (the Earth's surface); (d) is the vertical velocity $V(\mathrm{~cm} / \mathrm{s})$ of the Earth's surface. The carrier LF frequency is $\omega_{0}=5 \times 10^{4} \mathrm{~s}^{-1}$, the initial amplitude of the deformation of the LF wave is 0.01 ; the modulation frequency is $500 \mathrm{~s}^{-1}$, and the transverse scale is $\rho_{0}=0.5 \mathrm{~km}$.

on the nonlinear evolution of the seismic acoustic burst-like envelope of a finite transverse scale in the cylindrical coordinate frame. This method makes possible to prepare a simple modeling of Eqs. (5) and (6).

For the $\rho$-component we obtain the following equation:

$\frac{\partial^{2} U_{\rho}}{\partial \eta^{2}} \cdot\left(1-\frac{S_{t}^{2}}{S_{l}^{2}}\right) \cong-\frac{S_{l}^{2}-S_{t}^{2}}{S_{l}} \cdot \frac{\partial^{2} U_{z}}{\partial \rho \partial \eta}$

or

$\frac{\partial U_{\rho}}{\partial \eta} \cong-S_{1} \frac{\partial U_{z}}{\partial \rho}$.

Equation (7) shows that the $z$-component is very significant, the transverse profile is really smooth, so in our modeling it is possible to exclude the transverse component.
For the $z$-component we have:

$$
\begin{aligned}
& \frac{\partial U_{z}}{\partial z}-\frac{\Gamma(z)}{2 S_{l}^{3}} \cdot \frac{\partial^{2} U_{z}}{\partial \eta^{2}}+\frac{B(z)}{2 S_{l}^{4}} \cdot\left(\frac{\partial U_{z}}{\partial \eta}\right)^{2} \\
& -\frac{S_{l}}{2} \cdot \Delta_{\perp} \int_{-\alpha}^{\eta} U_{z}\left(\eta^{\prime}\right) \cdot d \eta^{\prime}=0 .
\end{aligned}
$$

This is the equation of Khokhlov-Zabolotskaya (Rudenko, 1995).

For numerical simulations, it is necessary to apply the fast Fourier transform with respect to the variable $\eta$. For a better approximation, it is possible to subdivide the Eq. (8) into LF and ELF parts of the spectrum. The classification of waves 


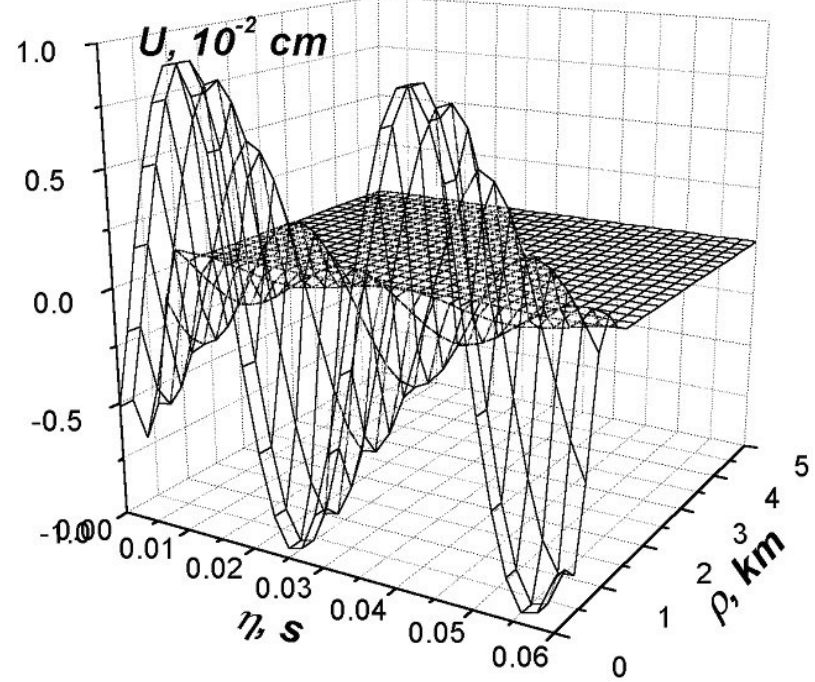

(a)

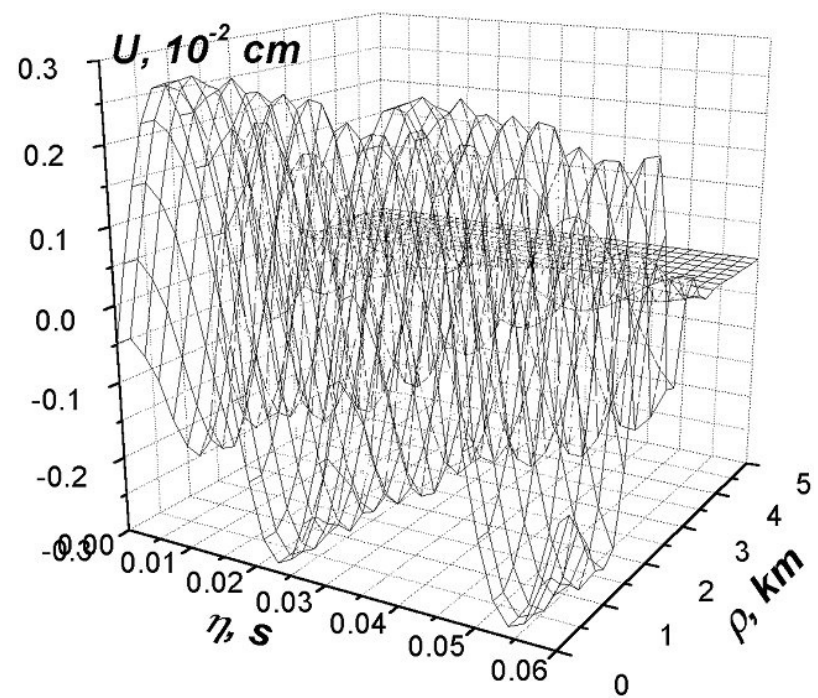

(c)

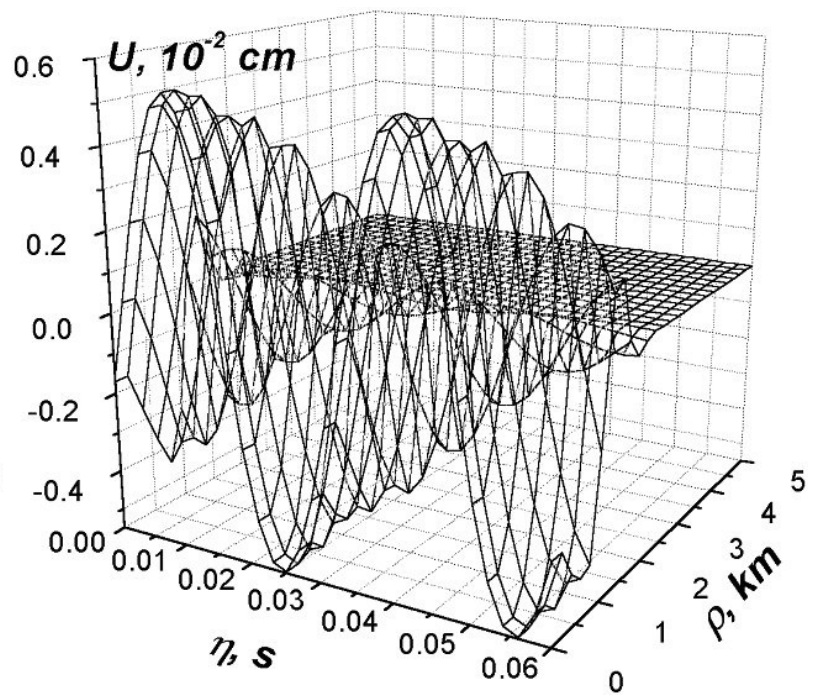

(b)

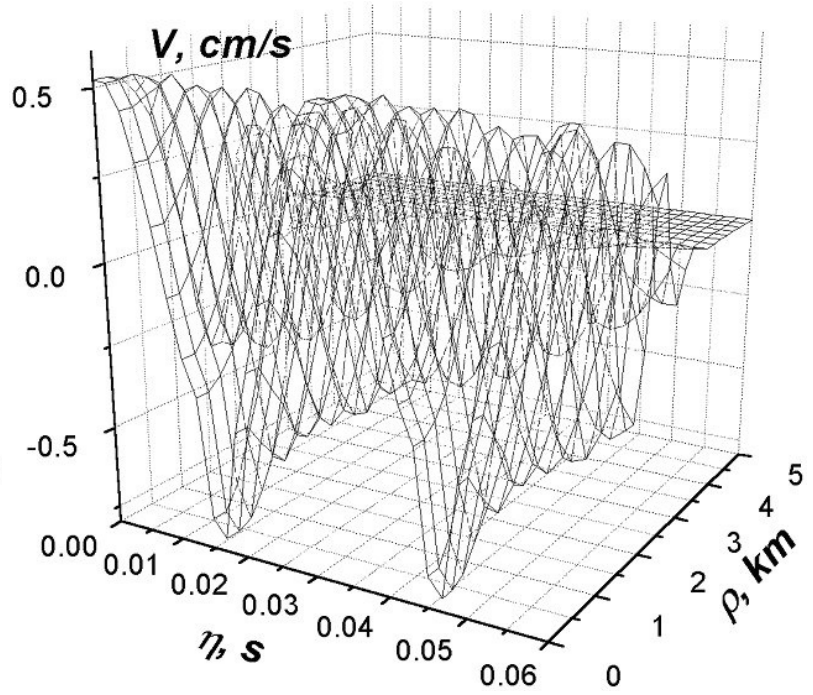

(d)

Fig. 5. The evolution of the nonlinearly excited ELF component of the mechanical displacement $U[\mathrm{~cm}]$ in the lithosphere: (a) at $z=5 \mathrm{~km}$, (b) at $z=10 \mathrm{~km}$; (c) at $z=20 \mathrm{~km}$ (the Earth's surface); (d) is the vertical velocity $V(\mathrm{~cm} / \mathrm{s})$ of the Earth's surface. The carrier LF frequency is $\omega_{0}=5 \times 10^{4} \mathrm{~s}^{-1}$, the initial amplitude of the deformation of the LF wave is 0.01 ; the modulation frequency is $100 \mathrm{~s}^{-1}$, the transverse scale is $\rho_{0}=0.5 \mathrm{~km}$.

is the following: the LF waves correspond to the frequency range of $f<100 \mathrm{kHz}$, ELF of $f<300 \mathrm{~Hz}$ and ULF $f<5 \mathrm{~Hz}$.

In simulations, the value of dissipation of ultrasonic waves in the lithosphere has been taken as $10 \mathrm{~km}^{-1}$, at the frequency $\omega=10^{6} \mathrm{~s}^{-1}$. It is assumed that the dissipation is proportional to $\omega^{2}$. The quadratic nonlinear module $B$ is about $B=10 S_{l}^{2}$, and the longitudinal sound velocity is $S_{l}=5 \mathrm{~km} / \mathrm{s}$. The results of the simulations are tolerant to some changes of the chosen parameters.

We take into account the diffraction only for the case of the ELF part. The results of the simulations are shown in Figs. 4 and 5. The VLF acoustic wave has the same amplitude of the elastic deformation, $10^{-2}$; the carrier frequency is $\omega_{0}=4 \times 10^{5} \mathrm{~s}^{-1}$. But in the Fig. 4 the ELF modulation frequency is $500 \mathrm{~s}^{-1}$; in the Fig. 5 this frequency is $100 \mathrm{~s}^{-1}$.
The LF acoustic waves present high losses, the essential feature of the nonlinearity, but do not present the wave diffraction. Correspondingly, the ELF acoustic waves are subject to both nonlinearity and the wave diffraction.

The Figs. 4, 5 illustrate that only the ELF waves (10$300 \mathrm{~Hz}$ ) pass through the Earth's lithosphere without essential losses and reach the Earth's surface. The acoustic waves at upper frequencies $(f>1000 \mathrm{~Hz}$ ) present high losses, whereas a nonlinear excitation of the ULF acoustic waves within the lithosphere is not effective and the ULF acoustic waves are subject to strong diffraction. The LF part dissipates very fast. 
3.2 Nonlinear passage through the atmosphere and the ionosphere

The theory of different effects caused by the acoustic channel is based on the hydrodynamic theory and the Maxwell's equations. We apply this theory to the nonlinear effects caused by the seismic waves. The ELF atmospheric acoustic waves interact in the atmosphere and the ionosphere and excite the ULF atmospheric acoustic waves, which reach the ionospheric altitudes. During earthquakes, the spectrum of the seismic waves is wide but only the ELF and ULF acoustic waves can reach the E- layer of the ionosphere. During the vertical propagation of ELF acoustic waves, also nonlinear frequency down-conversion into the ULF acoustic waves takes place. Here we are considering the processes associated with the propagation of fast acoustic and acoustic-gravity waves. A penetration of slow internal gravity waves to middle and upper ionosphere heights $(300-500 \mathrm{~km})$ has been considered in numerous papers (see, for instance, Gokhberg et al., 1994; Mareev et al., 2002).

For the acoustic channel of the lithosphere-ionosphere coupling, the above-mentioned circumstances are important. A nonlinear propagation of acoustic waves in the atmosphere is described by the following set of the hydrodynamic equations:

$\rho_{0}\left(\frac{\partial V_{z}}{\partial t}+v_{z} \frac{\partial V_{z}}{\partial z}\right)+\rho^{\prime} \frac{\partial V_{z}}{\partial t} \approx-\frac{\partial p^{\prime}}{\partial z}+\frac{\partial}{\partial z}\left(\rho_{0} v(z) \frac{\partial V_{z}}{\partial z}\right)-\rho^{\prime} g ;$

$\rho_{0} \frac{\partial V_{\perp}}{\partial t} \approx-\nabla_{\perp} p^{\prime}$

$\frac{\partial \rho^{\prime}}{\partial t}+\frac{\partial}{\partial z}\left(\rho_{0} V_{z}\right)+\rho_{0} \operatorname{div} V_{\perp}+\frac{\partial}{\partial z}\left(\rho^{\prime} V_{z}\right) \approx 0 ;$

$\frac{\partial p^{\prime}}{\partial t}-\rho_{0} g V_{z}+V_{z} \frac{\partial p^{\prime}}{\partial z} \approx-C_{s}^{2}\left(\rho_{0} \frac{V_{z}}{\partial z}+\rho_{0} d i v V_{\perp}+\gamma \rho^{\prime} \frac{\partial V_{z}}{\partial z}\right)$

Here $C_{s}$ is the sound velocity in air, $v(z) \approx v(0) \exp (-z / 2 H)$ is the kinematic viscosity of the air, $\rho, p$ are its total density and pressure, $\rho=\rho_{0}+\rho^{\prime}, p=p_{0}+p^{\prime} ; \rho_{0}, p_{0}$ are stationary values of atmospheric density and pressure $\left(\rho_{0}\right.$, $p_{0} \sim \exp (-z / H), H=k_{B} \mathrm{~T} / \mathrm{mg}, k_{B}$ is the Boltzmann's constant, and $m$ is the neutral gas mass of the ionosphere), $\left(V_{x}, V_{y}, V_{z}\right)$ are the components of the air velocity with $V_{\perp}=\left(V_{x}, V_{y}\right) ; \rho^{\prime}, p^{\prime}$ are the alternating parts and $g$ is the free fall acceleration.

We use the adiabatic equation for pressure with the adiabatic constant $\gamma$. It is assumed that nonlinearity is moderate and only quadratic nonlinear terms are preserved in the Eqs. (9). Note that all mechanisms of nonlinearity are essential. Also, we assume that the acoustic wave moves preferentially vertically upwards, and inequalities $\left|v_{x, y}\right| \ll v_{z}$ are valid. In a linear approximation, when viscosity is neglected, from Eqs. (9) we get for the acoustic wave the following velocity expression: $|\boldsymbol{V}(z, t)| \approx \exp (z / 2 H) \cdot \exp (i(\Omega t-K z))$, $K$ is the acoustic wave number, and the energy flux is $\rho_{0}(z)|\boldsymbol{V}(z, t)|^{2}=$ const. The numerical simulation confirms this analysis. Because the wave acoustic dispersion is small $\Omega>0.1 \mathrm{~s}^{-1}$ ), a number of harmonics are excited under the nonlinear interaction.
A slowly varying profile approach is suitable for the analysis of the nonlinear acoustic propagation in the atmosphere. It is possible to derive a single equation for the vertical component of the velocity of the air $v_{z}$. A dependence on only one transverse coordinate $\rho=\left(x^{2}+y^{2}\right)^{1 / 2}$ is assumed $(\partial / \partial \varphi=0)$.

When introducing new variables $V=V_{z} \exp (-z / 2 H), z$, $\eta=t-z / C_{s}$, and $\rho$, the resulting equation is:

$\frac{\partial}{\partial \eta}\left(\frac{\partial V}{\partial z}-\frac{\nu(0) e^{\frac{z}{H}}}{2 C_{s}^{3}} \frac{\partial^{2} V}{\partial \eta^{2}}-\frac{1+\gamma}{2} \frac{e^{\frac{z}{2 H}}}{2 C_{s}^{2}} \frac{\partial}{\partial \eta}\left(V^{2}\right)\right)=\frac{C_{s}}{2} \Delta_{\perp} V$.

Equation (10) is the Khokhlov-Zabolotskaya equation for a medium with a density decreasing along the z-axis. Here we consider the case when the envelope frequency is $\Omega \sim 1$ $\mathrm{s}^{-1} \gg \Omega_{a}, \Omega_{a}=C_{s} / 2 H$. After changing the independent variable $z: w=\exp (z / 2 H)$, it is possible to rewrite Eq. (10) as:

$$
\begin{aligned}
& \frac{\partial}{\partial \eta}\left(\frac{\partial V}{\partial w}-\frac{\nu(0) H w}{C_{s}^{3}} \frac{\partial^{2} V}{\partial \eta^{2}}-\frac{1+\gamma}{2} \frac{H}{C_{s}^{2}} \frac{\partial}{\partial \eta}\left(V^{2}\right)\right)=\frac{C_{s} H}{w} \Delta_{\perp} V \\
& V(w=1, t, \rho)=V_{0} \sin \left(\omega_{0} t\right) \exp \left(-\left(t / t_{0}\right)^{2}\right) \cdot \exp \left(-\left(\rho / \rho_{0}\right)^{2}\right) .
\end{aligned}
$$

The input ELF wave is burst-like, namely, it is a wave packet with a low-frequency carrier frequency $\omega_{0}$; its envelope lies in the ULF frequency range. The value of $\rho_{0}$ determines a transverse scale of the initial pulse.

After an excitation on the Earth's surface, an ELF wave is subject to nonlinearity that leads to the generation of higher harmonics and down-conversion, namely, increasing the ULF components. More than 100 harmonics are excited. The growth of higher harmonics leads to the creation of a saw-tooth like structure. This structure dissipates due to the viscosity. The ULF part of the spectrum is not subject to dissipation, but it increases due to this nonlinear interaction. When the transverse scale $\rho_{0} \sim 1-10 \mathrm{~km}$, the diffraction is not essential for the ELF wave but it can decrease the peak amplitudes of the ULF part. The results of the numerical simulations are analogous to the nonlinear propagation of acoustic waves in the lithosphere, but the frequency range is lower and the vertical scales are greater (see Grimalsky et al., 2003). The simulations have shown that there is an effective nonlinear interaction of the ELF and ULF atmospheric acoustic waves and a nonlinear excitation of the ULF atmosphereionosphere waves caused by the ELF seismic acoustic bursts on the surface of the Earth.

The nonlinear interaction of atmospheric acoustic waves additionally demonstrates the importance of the acoustic channel through the example of the nonlinear mechanism of the energy flux from the lithosphere into the ionosphere. The efficiency of the nonlinear acoustic transformation is quite high. For the initial ELF wave amplitude $V_{z}(z=0)=V_{0}=2.5 \mathrm{~cm} / \mathrm{s}$, the ULF wave amplitude is $V_{z} \equiv V(0) \exp (z / 2 H) \approx 300 \mathrm{~cm} / \mathrm{s}$ at the altitude $z=130 \mathrm{~km}$.

\section{Conclusions}

The modeling of seismo-electromagnetic phenomena caused by fracturing piezoelectric in rocks is studied. 
a) We consider a piezoelectric crystal of symmetry 32 under mechanical stresses, which produce a uniform crack motion. Further non-stationary mechanical stresses in the vicinity of the moving crack give rise to non-stationary polarization currents. These currents generate electromagnetic waves. The first kind of current is an electric polarization current $I_{P}$, and the second one, is the effective magnetization current $I_{M} \gg I_{P}$. This explains the spectra of electromagnetic waves caused by volcano, seismic, and impact events, namely, radio waves in the case of impact events (at frequencies $f<1 \mathrm{GHz}$ ), LF and $\operatorname{VLF}(f<100 \mathrm{kHz})$ and ELF $(f<300 \mathrm{~Hz})$ in the case of volcano and seismic activity. The obtained amplitude-frequency characteristics of seismo-electromagnetic waves, generated by fracturing piezoelectrics in rocks, confirm this analysis.

b) The simulation of the nonlinear passage of acoustic waves caused by underground fracturing, through the lithosphere, the atmosphere, and the ionosphere, shows that there is an effective nonlinear interaction of the LF and VLF bursts in the lithosphere. As a result of the nonlinear downconversion, the ELF waves emerge through the Earth's surface. Then the ELF acoustic waves move upwards in the atmosphere, and a nonlinear excitation of the ULF waves $(f<5 \mathrm{~Hz})$ takes place. The diffraction decreases the value of the pulse of the ULF waves under the passage through the atmosphere, but the losses of the ULF waves are small. We have in fact found that, after the passage through the lithosphere, there is a transformation of the LF seismic burst into ELF acoustic waves, which are registered in seismographs. In the ionosphere, a nonlinear frequency conversion of ELF waves into the ULF waves takes place. Thus, in addition, the nonlinear interaction of acoustic bursts shows the importance of the acoustic channel of lithosphere-ionosphere coupling.

Acknowledgements. The authors are grateful to two anonymous referees for questions and remarks that have given us the possibility to improve the text of the manuscript.

Edited by: M. Contadakis

Reviewed by: two referees

\section{References}

Aki, K. and Richards, P. G.: Quantitative seismology, W. H. Freeman and Comp., San Francisco, 1980.

Berezhnoy, A. A., Bervalds, E., Khavroshkin, O. B., Kovalenko, A. V., Ozolins, G., Paupere, M., Smirnov, G. T., and Tsyplakov, V. V.: Radio observations of the Moon during the activity periods of Leonid and Lyrid meteor streams, Baltic Astronomy, 11, 507527, 2002.

Galperin, Yu. I.: Aureol-3 results on ELF/VLF noises and Alfven waves induced by ground-level chemical explosions: a natural model of seismo-magnetospheric effects, in: Electromagnetic Phenomena Related to Earthquake Prediction, edited by: Hayakawa, M. and Fujinawa, Y., Tokyo, TERRAPUB, 349-359, 1994.

Gokhberg, M., Morgounov, V., and Pokhotelov, O.: Earthquake Prediction: Seismo-Electromagnetic Phenomena, Gordon and Breach, London, 280, 1995.
Gokhberg, M. B., Nekrasov, A. K., and Shalimov, S. L.: A new approach to the problem of the lithosphere-ionosphere coupling before the earthquakes, in: Electromagnetic Phenomena Related to Earthquake Prediction, edited by: Hayakawa, M. and Fujinawa, Y., Tokyo, TERRAPUB, 619-625, 1994.

Grimalsky, V., Koshevaya, S., Perez-Enriquez, R., and Kotsarenko, A.: Nonlinear Excitation of ULF Atmosphere-Ionosphere Waves and Magnetic Perturbations Caused by ELF Seismic Acoustic Bursts, Physica Scripta, 67, 453-456, 2003.

Hayakawa, M. and Molchanov, O. (Eds.): Seismo - Electromagnetics (Lithosphere - Atmosphere - Ionosphere Coupling), TERRAPUB, 335-343, 2002.

Hines, C. O.: The upper atmosphere in motion: A selection of papers with annotation, Geophys. Monogr. 18, AGU, Washington, D.C., 1974

Khavroshkin, O. B., Tsyplakov, V. V., and Berezhnoi, A. A.: Modification of the Moon's Microwave Radiation by the Fall of Lunar Prospector, Doklady Russian Acad. Sci., Earth Sciences, 376, 1, 90-92, 2001.

Kotsarenko, N., Perez Enriquez, R., and Koshevaya, S.: Excitation of plasma waves in the ionosphere caused by atmospheric acoustic waves, Astrophysics and Space Science, 246, 211-217, 1997.

Landau, L. D. and Lifshitz, E. M.: Electrodynamics of Continuous Media, Pergamon, London, 416, 1960.

Makarets, M. V., Koshevaya, S. V., and Gernets, A. A.: Electromagnetic Emission Caused by the Fracturing of Piezoelectrics in Rocks, Physica Scripta, 65, 268-272, 2002.

Mareev, E. A., Iudin, D. I., and Molchanov, O. A.: Mosaic source of internal gravity waves associated with seismic activity, in: Seismo-Electromagnetics (Lithosphere - Atmosphere - Ionosphere Coupling), edited by: Hayakawa, M. and Molchanov, O., TERRAPUB, Tokyo, 335-342, 2002.

Maugin, G. A.: Continuum Mechanics of Electromagnetic Solids, North-Holland, Amsterdam, 598, 1988.

Molchanov, O. A., Hayakawa, M., and Rafalsky, V. A.: Penetration characteristics of electromagnetic emissions from an underground seismic source into the atmosphere, ionosphere, and magnetosphere, J. Geophys. Res., 100, 1691-1712, 1995.

Molchanov, O. A. and Hayakawa, M.: On the generation mechanism of ULF seismogenic electromagnetic emissions, Phys. Earth Planet. Inter., 105, 201-210, 1998.

Molchanov, O. A., Kulchitsky, A. V., and Hayakawa, M.: Inductive seismo-electromagnetic effect in relation to seismogenic ULF emission, Nat. Haz. Earth. Sys. Sci., 1, 61-67, 2001,

SRef-ID: 1684-9981/nhess/2001-1-61.

Musatenko, S. I., Kurochka, E. V., Aslanyan, A. M., Borovkov, O. V., Gulyan, A. G., Ivchenko, V. N., Kotsarenko, A. N., Slipchenko, A. S., Margorin, O. K., and Nabatov, A. S.: Fluctuations of Jupiter radio emission at $3.6 \mathrm{~cm}$ due to the impact of the fragments of Comet Shoemaker-Levy, Kinematika i Fizika Nebesnykh Tel, 13,1, 64-73, 1997.

Rudenko, O.: Nonlinear Sawtooth-Shaped Waves, Phys.-Uspekhi, 38, 9, 965-989, 1995

Surkov, V. V., Molchanov, O. A., and Hayakawa, M.: Preearthquake ULF electromagnetic perturbations as a result of inductive seismomagnetic phenomena during microfracturing, $\mathrm{J}$. Atmos. Sol-Ter. Phys., 65, 31-46, 2003.

Tarantsev, A. and Birfeld, Ya.: The Brief Descriptions of Discoveries. CNIIPII (USSR, Moscow), Discovery No. 128, 1973.

Warwick, J. W.: Radio Astronomical and Satellite Studies of the Atmosphere. Edited by Aarons, J., Amsterdam, North-Holland, 400, 1963. 